\title{
Optimal bounds for the first and second Seiffert means in terms of geometric, arithmetic and contraharmonic means
}

Yu-Ming Chu ${ }^{1 *}$, Wei-Mao Qian², Li-Min Wu³ and Xiao-Hui Zhang ${ }^{1}$

\section{"Correspondence:}

chuyuming2005@126.com

'school of Mathematics and

Computation Sciences, Hunan City

University, Yiyang, 413000, China

Full list of author information is

available at the end of the article

\begin{abstract}
In this paper, we find the greatest values $\alpha, \lambda$ and the least values $\beta, \mu$ such that the double inequalities $\alpha[G(a, b) / 3+2 A(a, b) / 3]+(1-\alpha) G^{1 / 3}(a, b) A^{2 / 3}(a, b)<P(a, b)<$ $\beta[G(a, b) / 3+2 A(a, b) / 3]+(1-\beta) G^{1 / 3}(a, b) A^{2 / 3}(a, b)$ and $\lambda[C(a, b) / 3+2 A(a, b) / 3]+$ $(1-\lambda) C^{1 / 3}(a, b) A^{2 / 3}(a, b)<T(a, b)<\mu[C(a, b) / 3+2 A(a, b) / 3]+(1-\mu) C^{1 / 3}(a, b) A^{2 / 3}(a, b)$ hold for all $a, b>0$ with $a \neq b$. Here $G(a, b), A(a, b), C(a, b), P(a, b)$ and $T(a, b)$ denote the geometric, arithmetic, contraharmonic, first Seiffert and second Seiffert means of two positive numbers $a$ and $b$, respectively.
\end{abstract}

MSC: $26 \mathrm{E} 60$

Keywords: first Seiffert mean; second Seiffert mean; geometric mean; arithmetic mean; contraharmonic mean

\section{Introduction}

For $a, b>0$ with $a \neq b$, the first and second Seiffert means $P(a, b)[1]$ and $T(a, b)[2]$ are defined by

$$
P(a, b)=\frac{a-b}{4 \arctan (\sqrt{a / b})-\pi}
$$

and

$$
T(a, b)=\frac{a-b}{2 \arctan [(a-b) /(a+b)]},
$$

respectively.

Recently, both means $P$ and $T$ have been the subject of intensive research. In particular, many remarkable inequalities for $P$ and $T$ can be found in the literature [3-9]. The first Seiffert mean $P(a, b)$ can be rewritten as (see [10, Eq. (2.4)])

$$
P(a, b)=\frac{a-b}{2 \arcsin [(a-b) /(a+b)]} .
$$

Let $H(a, b)=2 a b /(a+b), G(a, b)=\sqrt{a b}, L(a, b)=(b-a) /(\log b-\log a), I(a, b)=$ $1 / e\left(b^{b} / a^{a}\right)^{1 /(b-a)}, A(a, b)=(a+b) / 2, Q(a, b)=\sqrt{\left(a^{2}+b^{2}\right) / 2}, C(a, b)=\left(a^{2}+b^{2}\right) /(a+b)$, 
$L_{r}(a, b)=\left(a^{r+1}+b^{r+1}\right) /\left(a^{r}+b^{r}\right)$, and $M_{r}(a, b)=\left[\left(a^{r}+b^{r}\right) / 2\right]^{1 / r}(r \neq 0)$ and $M_{0}(a, b)=G(a, b)$ be the harmonic, geometric, logarithmic, identric, arithmetic, quadratic, contraharmonic, $r$ th Lehmer and $r$ th power means of two distinct positive real numbers $a$ and $b$, respectively. Then both $L_{r}(a, b)$ and $M_{r}(a, b)$ are strictly increasing with respect to $r \in \mathbb{R}$ for fixed $a, b>0$ with $a \neq b$, and the inequalities

$$
\begin{aligned}
H(a, b) & =L_{-1}(a, b)=M_{-1}(a, b)<G(a, b)=L_{-1 / 2}(a, b) \\
& =M_{0}(a, b)<L(a, b)<P(a, b)<I(a, b)<A(a, b)=L_{0}(a, b) \\
& =M_{1}(a, b)<T(a, b)<Q(a, b)=M_{2}(a, b)<C(a, b)=L_{1}(a, b)
\end{aligned}
$$

hold for all $a, b>0$ with $a \neq b$.

Jagers [11] and Seiffert [2] proved that the inequalities

$$
M_{1 / 2}(a, b)<P(a, b)<M_{2 / 3}(a, b), \quad M_{1}(a, b)<T(a, b)<M_{2}(a, b)
$$

hold for $a, b>0$ with $a \neq b$.

Costin and Toader [12] proved that the double inequality

$$
M_{4 / 3}(a, b)<T(a, b)<M_{5 / 3}(a, b)
$$

holds for $a, b>0$ with $a \neq b$.

In [13-17], the authors proved that the inequalities

$$
\begin{array}{lc}
M_{p}(a, b)<P(a, b)<M_{q}(a, b), & M_{r}(a, b)<T(a, b)<M_{s}(a, b), \\
L_{\alpha}(a, b)<P(a, b)<L_{\beta}(a, b), & L_{\sigma}(a, b)<T(a, b)<L_{\tau}(a, b), \\
P(a, b)>\left[\frac{b^{\lambda}-a^{\lambda}}{\lambda(\log b-\log a)}\right]^{1 / \lambda}, & T(a, b)>\left[\frac{b^{\mu}-a^{\mu}}{\mu(\log b-\log a)}\right]^{1 / \mu}
\end{array}
$$

hold for $a, b>0$ with $a \neq b$ if and only if $p \leq \log \pi / \log 2, q \geq 2 / 3, r \leq \log 2 /(\log \pi-\log 2)$, $s \geq 5 / 3, \alpha \leq-1 / 6, \beta \geq 0, \sigma \leq 0, \tau \geq 1 / 3, \lambda \geq 2$ and $\mu \geq 5$.

Gao [18] proved that $\alpha=e / \pi, \beta=1, \lambda=1$ and $\mu=2 e / \pi$ are the best possible constants such that the double inequalities

$$
\alpha I(a, b)<P(a, b)<\beta I(a, b), \quad \lambda I(a, b)<T(a, b)<\mu I(a, b)
$$

hold for $a, b>0$ with $a \neq b$.

In $[19,20]$, the authors proved that the double inequalities

$$
\begin{aligned}
& \alpha_{1} C(a, b)+\left(1-\alpha_{1}\right) G(a, b)<P(a, b)<\beta_{1} C(a, b)+\left(1-\beta_{1}\right) G(a, b), \\
& \alpha_{2} A(a, b)+\left(1-\alpha_{2}\right) G(a, b)<P(a, b)<\beta_{2} A(a, b)+\left(1-\beta_{2}\right) G(a, b), \\
& \alpha_{3} C(a, b)+\left(1-\alpha_{3}\right) H(a, b)<T(a, b)<\beta_{3} C(a, b)+\left(1-\beta_{3}\right) H(a, b)
\end{aligned}
$$

hold for $a, b>0$ with $a \neq b$ if and only if $\alpha_{1} \leq 2 / 9, \beta_{1} \geq 1 / \pi, \alpha_{2} \leq 2 / \pi, \beta_{2} \geq 2 / 3, \alpha_{3} \leq 2 / \pi$ and $\beta_{3} \geq 2 / 3$. 
Let $p \geq 1 / 2, q \geq 1, t_{1}, t_{2} \in(1 / 2,1)$ and $t_{3}, t_{4} \in(0,1 / 2)$. Then the authors in [21,22] proved that the double inequalities

$$
\begin{aligned}
& C^{p} {\left[t_{1} a+\left(1-t_{1}\right) b, t_{1} b+\left(1-t_{1}\right) a\right] A^{1-p}(a, b) } \\
&<T(a, b)<C^{p}\left[t_{2} a+\left(1-t_{2}\right) b, t_{2} b+\left(1-t_{2}\right) a\right] A^{1-p}(a, b), \\
& G^{q}\left[t_{3} a+\left(1-t_{3}\right) b, t_{3} b+\left(1-t_{3}\right) a\right] A^{1-q}(a, b) \\
& \quad<P(a, b)<G^{q}\left[t_{4} a+\left(1-t_{4}\right) b, t_{4} b+\left(1-t_{4}\right) a\right] A^{1-q}(a, b)
\end{aligned}
$$

hold for $a, b>0$ with $a \neq b$ if and only if $t_{1} \leq\left[1+\sqrt{(4 / \pi)^{1 / p}-1}\right] / 2, t_{2} \geq 1 / 2+\sqrt{3 p} /(6 p)$, $t_{3} \leq\left[1-\sqrt{1-(2 / \pi)^{2 / q}}\right] / 2$ and $t_{4} \geq(1-1 / \sqrt{3 q}) / 2$.

Yang et al. [23] proved that the double inequality

$$
\frac{Q^{2}(a, b)}{L_{p-1}(a, b)}<T(a, b)<\frac{Q^{2}(a, b)}{L_{q-1}(a, b)}
$$

holds for $a, b>0$ with $a \neq b$ if and only if $p \geq 5 / 3$ and $q \leq 1$.

Sándor [24] and Jiang et al. [25] proved that the inequalities

$$
\begin{aligned}
& G^{1 / 3}(a, b) A^{2 / 3}(a, b)<P(a, b)<\frac{1}{3} G(a, b)+\frac{2}{3} A(a, b) \\
& T(a, b)<\frac{1}{3} C(a, b)+\frac{2}{3} A(a, b)
\end{aligned}
$$

hold for $a, b>0$ with $a \neq b$.

In [26], Sándor found that $T(a, b)$ is the common limit of the sequences $\left\{u_{n}\right\}$ and $\left\{v_{n}\right\}$ given by

$$
u_{0}=A(a, b), \quad v_{0}=Q(a, b), \quad u_{n+1}=\frac{u_{n}+v_{n}}{2}, \quad v_{n+1}=\sqrt{u_{n+1} v_{n}} \quad(n \geq 0)
$$

and established a more general inequality

$$
\sqrt[3]{u_{n} v_{n}^{2}}<T(a, b)<\frac{u_{n}+2 v_{n}}{3}
$$

for all $n \geq 0$ and $a, b>0$ with $a \neq b$. In particular, let $n=0$, then (1.4) and (1.5) together with the identity $Q^{2 / 3}(a, b) A^{1 / 3}(a, b)=C^{1 / 3}(a, b) A^{2 / 3}(a, b)$ lead to

$$
C^{1 / 3}(a, b) A^{2 / 3}(a, b)<T(a, b)<\frac{1}{3} C(a, b)+\frac{2}{3} A(a, b)
$$

for all $a, b>0$ with $a \neq b$.

Motivated by inequalities (1.3) and (1.6), we naturally ask: what are the best possible parameters $\alpha, \beta, \lambda$ and $\mu$ such that the double inequalities

$$
\begin{aligned}
& \alpha\left[\frac{1}{3} G(a, b)+\frac{2}{3} A(a, b)\right]+(1-\alpha) G^{1 / 3}(a, b) A^{2 / 3}(a, b) \\
& <P(a, b)<\beta\left[\frac{1}{3} G(a, b)+\frac{2}{3} A(a, b)\right]+(1-\beta) G^{1 / 3}(a, b) A^{2 / 3}(a, b),
\end{aligned}
$$




$$
\begin{aligned}
\lambda[ & \left.\frac{1}{3} C(a, b)+\frac{2}{3} A(a, b)\right]+(1-\lambda) C^{1 / 3}(a, b) A^{2 / 3}(a, b) \\
& <T(a, b)<\mu\left[\frac{1}{3} C(a, b)+\frac{2}{3} A(a, b)\right]+(1-\mu) C^{1 / 3}(a, b) A^{2 / 3}(a, b)
\end{aligned}
$$

hold for all $a, b>0$ with $a \neq b$ ? The purpose of this paper is to answer this question.

\section{Lemmas}

In order to establish our main results, we need two lemmas, which we present in this section.

Lemma 2.1 Let $g(t)=-p^{2} t^{6}-2 p^{2} t^{5}+3\left(p^{2}-4 p+2\right) t^{4}+2\left(2 p^{2}-9 p+6\right) t^{3}-\left(4 p^{2}+6 p-\right.$ $9) t^{2}+6(1-p) t+3(1-p)$. Then the following statements are true:

(1) If $p=4 / 5$, then $g(t)>0$ for all $t \in(0,1)$.

(2) If $p=3 / \pi$, then there exists $\lambda_{0} \in(0,1)$ such that $g(t)>0$ for $t \in\left(0, \lambda_{0}\right)$ and $g(t)<0$ for $t \in\left(\lambda_{0}, 1\right)$.

Proof Part (1) follows easily from

$$
g(t)=\frac{1}{25}(1-t)\left(16 t^{5}+48 t^{4}+90 t^{3}+86 t^{2}+45 t+15\right)>0
$$

for all $t \in(0,1)$ if $p=4 / 5$.

For part (2), if $p=3 / \pi$, then numerical computations lead to

$$
\begin{aligned}
& p^{2}-4 p+2=\frac{2 \pi^{2}-12 \pi+9}{\pi^{2}}<0, \\
& 2 p^{2}-9 p+6=\frac{6 \pi^{2}-27 \pi+18}{\pi^{2}}<0, \\
& 4 p^{2}+6 p-9=\frac{-9 \pi^{2}+18 \pi+36}{\pi^{2}}>0, \\
& g(0)= \frac{3(\pi-3)}{\pi}>0, \\
& g(1)= \frac{9(4 \pi-15)}{\pi}<0, \\
& g^{\prime}(t)=-6 p^{2} t^{5}-10 p^{2} t^{4}+12\left(p^{2}-4 p+2\right) t^{3} \\
&+6\left(2 p^{2}-9 p+6\right) t^{2}-2\left(4 p^{2}+6 p-9\right) t+6(1-p), \\
& g^{\prime}(0)= \frac{6(\pi-3)}{\pi}>0, \\
& g^{\prime}(1)= \frac{84 \pi-360}{\pi}<0, \\
& g^{\prime \prime}(t)=-30 p^{2} t^{4}-40 p^{2} t^{3}+36\left(p^{2}-4 p+2\right) t^{2} \\
&+12\left(2 p^{2}-9 p+6\right) t-2\left(4 p^{2}+6 p-9\right) .
\end{aligned}
$$

It follows from (2.1)-(2.3) and (2.8) that $g^{\prime}$ is strictly decreasing on $(0,1)$. Then (2.6) and (2.7) lead to the conclusion that there exists $\lambda_{1} \in(0,1)$ such that $g$ is strictly increasing on $\left(0, \lambda_{1}\right]$ and strictly decreasing on $\left[\lambda_{1}, 1\right)$. 
Therefore, part (2) follows from (2.4) and (2.5) together with the piecewise monotonicity of $g^{\prime}$.

Lemma 2.2 Let $h(t)=q(q+3) t^{4}+2 q(q+3) t^{3}-3\left(q^{2}-6 q+1\right) t^{2}-2\left(2 q^{2}-9 q+3\right) t+4 q^{2}$. Then the following statements are true:

(1) If $q=1 / 5$, then $h(t)>0$ for $t \in(1, \sqrt[3]{2})$.

(2) If $q=[3(\sqrt[3]{2} \pi-4)] /[(3 \sqrt[3]{2}-4) \pi]=0.1814 \ldots$, then there exists $\mu_{0} \in(1, \sqrt[3]{2})$ such that $h(t)<0$ for $t \in\left(1, \mu_{0}\right)$ and $h(t)>0$ for $t \in\left(\mu_{0}, \sqrt[3]{2}\right)$.

Proof Part (1) follows easily from

$$
h(t)=\frac{4(t-1)}{25}\left(4 t^{3}+12 t^{2}+15 t-1\right)>0
$$

for all $t \in(1, \sqrt[3]{2})$ if $q=1 / 5$.

For part (2), if $q=[3(\sqrt[3]{2} \pi-4)] /[(3 \sqrt[3]{2}-4) \pi]$, then numerical computations lead to

$$
\begin{aligned}
& q^{2}-6 q+1=-0.0556 \ldots<0, \\
& h(1)=9(5 q-1)=-0.836 \ldots<0, \\
& h(\sqrt[3]{2})=0.548 \ldots>0, \\
& h^{\prime}(t)=4 q(q+3) t^{3}+6 q(q+3) t^{2}-6\left(q^{2}-6 q+1\right) t-2\left(2 q^{2}-9 q+3\right) .
\end{aligned}
$$

It follows from (2.9) and (2.12) that

$$
\begin{aligned}
h^{\prime}(t) & >4 q(q+3)+6 q(q+3)-6\left(q^{2}-6 q+1\right)-2\left(2 q^{2}-9 q+3\right) \\
& =12(7 q-1)=3.239 \ldots>0
\end{aligned}
$$

for $t \in(1, \sqrt[3]{2})$.

Therefore, part (2) follows easily from (2.10) and (2.11) together with (2.13).

\section{Main results}

Theorem 3.1 The double inequality

$$
\begin{aligned}
\alpha[ & \left.\frac{1}{3} G(a, b)+\frac{2}{3} A(a, b)\right]+(1-\alpha) G^{1 / 3}(a, b) A^{2 / 3}(a, b) \\
& <P(a, b)<\beta\left[\frac{1}{3} G(a, b)+\frac{2}{3} A(a, b)\right]+(1-\beta) G^{1 / 3}(a, b) A^{2 / 3}(a, b)
\end{aligned}
$$

holds for all $a, b>0$ with $a \neq b$ if and only if $\alpha \leq 4 / 5$ and $\beta \geq 3 / \pi$.

Proof Firstly, we prove that the inequalities

$$
\begin{aligned}
& P(a, b)>\frac{4}{5}\left[\frac{1}{3} G(a, b)+\frac{2}{3} A(a, b)\right]+\frac{1}{5} G^{1 / 3}(a, b) A^{2 / 3}(a, b), \\
& P(a, b)<\frac{3}{\pi}\left[\frac{1}{3} G(a, b)+\frac{2}{3} A(a, b)\right]+\left(1-\frac{3}{\pi}\right) G^{1 / 3}(a, b) A^{2 / 3}(a, b)
\end{aligned}
$$

hold for all $a, b>0$ with $a \neq b$. 
Since $P(a, b), A(a, b)$ and $G(a, b)$ are symmetric and homogenous of degree 1, without loss of generality, we assume that $a>b$. Let $x=(a-b) /(a+b) \in(0,1)$ and $p \in(0,1)$. Then (1.2) leads to

$$
\begin{aligned}
& \frac{P(a, b)}{A(a, b)}=\frac{x}{\arcsin (x)}, \quad \frac{G(a, b)}{A(a, b)}=\sqrt{1-x^{2}}, \\
& \frac{P(a, b)-G^{1 / 3}(a, b) A^{2 / 3}(a, b)}{G(a, b) / 3+2 A(a, b) / 3-G^{1 / 3}(a, b) A^{2 / 3}(a, b)} \\
& =\frac{x / \arcsin (x)-\sqrt[6]{1-x^{2}}}{2 / 3+\sqrt{1-x^{2}} / 3-\sqrt[6]{1-x^{2}}}, \\
& \lim _{x \rightarrow 0^{+}} \frac{x / \arcsin (x)-\sqrt[6]{1-x^{2}}}{2 / 3+\sqrt{1-x^{2}} / 3-\sqrt[6]{1-x^{2}}}=\frac{4}{5}, \\
& \lim _{x \rightarrow 1^{-}} \frac{x / \arcsin (x)-\sqrt[6]{1-x^{2}}}{2 / 3+\sqrt{1-x^{2}} / 3-\sqrt[6]{1-x^{2}}}=\frac{3}{\pi}, \\
& P(a, b)-p\left[\frac{1}{3} G(a, b)+\frac{2}{3} A(a, b)\right]-(1-p) G^{1 / 3}(a, b) A^{2 / 3}(a, b) \\
& \quad=A(a, b)\left[\frac{x}{\arcsin (x)}-p\left(\frac{1}{3} \sqrt{1-x^{2}}+\frac{2}{3}\right)-(1-p) \sqrt[6]{1-x^{2}}\right] \\
& \quad=\frac{A(a, b)\left[p\left(2+\sqrt{1-x^{2}}\right)+3(1-p) \sqrt[6]{1-x^{2}}\right]}{3 \arcsin (x)} G(x),
\end{aligned}
$$

where

$$
\begin{aligned}
& G(x)=\frac{3 x}{p\left(2+\sqrt{1-x^{2}}\right)+3(1-p) \sqrt[6]{1-x^{2}}}-\arcsin (x), \\
& G(0)=0 \\
& G(1)=\frac{3}{2 p}-\frac{\pi}{2}, \\
& G^{\prime}(x)=\frac{\left(1-\sqrt[6]{1-x^{2}}\right)^{2}}{\sqrt[6]{\left(1-x^{2}\right)^{5}}\left[p\left(2+\sqrt{1-x^{2}}\right)+3(1-p) \sqrt[6]{1-x^{2}}\right]^{2}} g\left(\sqrt[6]{1-x^{2}}\right),
\end{aligned}
$$

where the function $g(\cdot)$ is defined as in Lemma 2.1.

We divide the proof into two cases.

Case 1. $p=4 / 5$. Then (3.1) follows easily from (3.6), (3.7), (3.9) and Lemma 2.1(1).

Case 2. $p=3 / \pi$. Then Lemma 2.1(2) and (3.9) lead to the conclusion that there exists $x_{0} \in(0,1)$ such that $G$ is strictly decreasing on $\left(0, x_{0}\right]$ and strictly increasing on $\left[x_{0}, 1\right)$.

Note that (3.8) becomes

$$
G(1)=0
$$

It follows from (3.7) and (3.10) together with the piecewise monotonicity of $G$ that

$$
G(x)<0
$$

for all $x \in(0,1)$. 
Therefore, (3.2) follows from (3.6) and (3.11), and Theorem 3.1 follows from (3.1) and (3.2) in conjunction with the following statements.

- If $\alpha>4 / 5$, then equations (3.3) and (3.4) lead to the conclusion that there exists $0<\delta_{1}<1$ such that $P(a, b)<\alpha[G(a, b) / 3+2 A(a, b) / 3]+(1-\alpha) G^{1 / 3}(a, b) A^{2 / 3}(a, b)$ for all $a, b>0$ with $(a-b) /(a+b) \in\left(0, \delta_{1}\right)$.

- If $\beta<3 / \pi$, then equations (3.3) and (3.5) imply that there exists $0<\delta_{2}<1$ such that $P(a, b)>\beta[G(a, b) / 3+2 A(a, b) / 3]+(1-\beta) G^{1 / 3}(a, b) A^{2 / 3}(a, b)$ for all $a, b>0$ with $(a-b) /(a+b) \in\left(1-\delta_{2}, 1\right)$.

Theorem 3.2 The double inequality

$$
\begin{aligned}
\lambda[ & \left.\frac{1}{3} C(a, b)+\frac{2}{3} A(a, b)\right]+(1-\lambda) C^{1 / 3}(a, b) A^{2 / 3}(a, b) \\
& <T(a, b)<\mu\left[\frac{1}{3} C(a, b)+\frac{2}{3} A(a, b)\right]+(1-\mu) C^{1 / 3}(a, b) A^{2 / 3}(a, b)
\end{aligned}
$$

holds for all $a, b>0$ with $a \neq b$ if and only if $\lambda \leq[3(\sqrt[3]{2} \pi-4)] /[(3 \sqrt[3]{2}-4) \pi]=0.1814 \ldots$ and $\mu \geq 1 / 5$.

Proof Let $\lambda^{*}=[3(\sqrt[3]{2} \pi-4)] /[(3 \sqrt[3]{2}-4) \pi]$. Firstly, we prove that the inequalities

$$
\begin{aligned}
& T(a, b)<\frac{1}{5}\left[\frac{1}{3} C(a, b)+\frac{2}{3} A(a, b)\right]+\frac{4}{5} C^{1 / 3}(a, b) A^{2 / 3}(a, b), \\
& T(a, b)>\lambda^{*}\left[\frac{1}{3} C(a, b)+\frac{2}{3} A(a, b)\right]+\left(1-\lambda^{*}\right) C^{1 / 3}(a, b) A^{2 / 3}(a, b)
\end{aligned}
$$

hold for all $a, b>0$ with $a \neq b$.

Since $T(a, b), A(a, b)$ and $C(a, b)$ are symmetric and homogenous of degree 1 , without loss of generality, we assume that $a>b$. Let $x=(a-b) /(a+b) \in(0,1)$ and $q \in(0,1)$. Then (1.1) leads to

$$
\begin{aligned}
& \frac{T(a, b)}{A(a, b)}=\frac{x}{\arctan (x)}, \quad \frac{C(a, b)}{A(a, b)}=1+x^{2}, \\
& \frac{T(a, b)-C^{1 / 3}(a, b) A^{2 / 3}(a, b)}{C(a, b) / 3+2 A(a, b) / 3-C^{1 / 3}(a, b) A^{2 / 3}(a, b)} \\
& =\frac{x / \arctan x-\sqrt[3]{1+x^{2}}}{2 / 3+\left(1+x^{2}\right) / 3-\sqrt[3]{1+x^{2}}}, \\
& \lim _{x \rightarrow 0^{+}} \frac{x / \arctan x-\sqrt[3]{1+x^{2}}}{2 / 3+\left(1+x^{2}\right) / 3-\sqrt[3]{1+x^{2}}}=\frac{1}{5}, \\
& \lim _{x \rightarrow 1^{-}} \frac{x / \arctan x-\sqrt[3]{1+x^{2}}}{2 / 3+\left(1+x^{2}\right) / 3-\sqrt[3]{1+x^{2}}}=\lambda^{*}, \\
& T(a, b)-q\left[\frac{1}{3} C(a, b)+\frac{2}{3} A(a, b)\right]-(1-q) C^{1 / 3}(a, b) A^{2 / 3}(a, b) \\
& \quad=A(a, b)\left[\frac{x}{\arctan (x)}-\frac{q}{3}\left(3+x^{2}\right)-(1-q) \sqrt[3]{1+x^{2}}\right] \\
& \quad=\frac{A(a, b)\left[q\left(3+x^{2}\right)+3(1-q) \sqrt[3]{1+x^{2}}\right]}{3 \arctan (x)} H(x),
\end{aligned}
$$


where

$$
\begin{aligned}
& H(x)=\frac{3 x}{q\left(3+x^{2}\right)+3(1-q) \sqrt[3]{1+x^{2}}}-\arctan (x), \\
& H(0)=0, \\
& H(1)=\frac{3}{4 q+3 \sqrt[3]{2}(1-q)}-\frac{\pi}{4}, \\
& H^{\prime}(x)=-\frac{\left(\sqrt[3]{1+x^{2}}-1\right)^{2}}{\left(1+x^{2}\right)\left[q\left(3+x^{2}\right)+3(1-q) \sqrt[3]{1+x^{2}}\right]^{2}} h\left(\sqrt[3]{1+x^{2}}\right),
\end{aligned}
$$

where the function $h(\cdot)$ is defined as in Lemma 2.2.

We divide the proof into two cases.

Case 1. $q=1 / 5$. Then (3.12) follows easily from Lemma 2.2(1), (3.17), (3.18) and (3.20).

Case 2. $q=\lambda^{*}$. Then Lemma 2.2(2) and (3.20) lead to the conclusion that there exists $x^{*} \in(0,1)$ such that $H$ is strictly increasing on $\left(0, x^{*}\right]$ and strictly decreasing on $\left[x^{*}, 1\right)$.

Note that (3.19) becomes

$$
H(1)=0 .
$$

Therefore, (3.13) follows from (3.17), (3.18), (3.21) and the piecewise monotonicity of $H$, and Theorem 3.2 follows from (3.12) and (3.13) in conjunction with the following statements.

- If $\mu<1 / 5$, then equations (3.14) and (3.15) lead to the conclusion that there exists $0<\delta_{3}<1$ such that $T(a, b)>\mu[C(a, b) / 3+2 A(a, b) / 3]+(1-\mu) C^{1 / 3}(a, b) A^{2 / 3}(a, b)$ for all $a, b>0$ with $(a-b) /(a+b) \in\left(0, \delta_{3}\right)$.

- If $\lambda>\lambda^{*}$, then equations (3.14) and (3.16) imply that there exists $0<\delta_{4}<1$ such that $T(a, b)<\lambda[C(a, b) / 3+2 A(a, b) / 3]+(1-\lambda) C^{1 / 3}(a, b) A^{2 / 3}(a, b)$ for all $a, b>0$ with $(a-b) /(a+b) \in\left(1-\delta_{4}, 1\right)$.

Competing interests

The authors declare that they have no competing interests.

Authors' contributions

All authors contributed equally to the writing of this paper. All authors read and approved the final manuscript.

\section{Author details}

'School of Mathematics and Computation Sciences, Hunan City University, Yiyang, 413000, China. ${ }^{2}$ School of Distance Education, Huzhou Broadcast and TV University, Huzhou, 313000, China. ${ }^{3}$ Department of Mathematics, Huzhou University, Huzhou, 313000, China.

\section{Acknowledgements}

The research was supported by the Natural Science Foundation of China under Grants 11401191, 11171307 and 61374086, and the Natural Science Foundation of Zhejiang Province under Grant LY13A010004.

Received: 30 October 2014 Accepted: 20 January 2015 Published online: 04 February 2015

\section{References}

1. Seiffert, H-J: Problem 887. Nieuw Arch. Wiskd. 11(2), 176 (1993)

2. Seiffert, H-J: Aufgabe $\beta 16$. Wurzel 29, 221-222 (1995)

3. Chu, Y-M, Hou, S-W: Sharp bounds for Seiffert mean in terms of contraharmonic mean. Abstr. Appl. Anal. 2012, Article ID 425175 (2012)

4. Chu, Y-M, Wang, M-K, Wang, Z-K: Best possible inequalities among harmonic, geometric, logarithmic and Seiffert means. Math. Inequal. Appl. 15(2), 415-422 (2012) 
5. Costin, I, Toader, G: A nice separation of some Seiffert-type means by power means. Int. J. Math. Math. Sci. 2012, Article ID 430692 (2012)

6. Jiang, W-D: Some sharp inequalities involving reciprocals of the Seiffert and other means. J. Math. Inequal. 6(4), 593-599 (2012)

7. Witkowski, A: Optimal weighted harmonic interpolations between Seiffert means. Colloq. Math. 130(2), 265-279 (2013)

8. Matejíčka, L: Sharp bounds for the weighted geometric mean of the first Seiffert and logarithmic means in terms of weighted generalized Heronian mean. Abstr. Appl. Anal. 2013, Article ID 721539 (2013)

9. Yang, Z-H: Sharp bounds for Seiffert mean in terms of weighted power means of arithmetic mean and geometric mean. Math. Inequal. Appl. 17(2), 499-511 (2014)

10. Neuman, E, Sándor, J: On the Schwab-Borchardt mean. Math. Pannon. 14(2), 253-266 (2003)

11. Jagers, AA: Solution of Problem 887. Nieuw Arch. Wiskd. 12, 230-231 (1994)

12. Costin, I, Toader, G: A separation of some Seiffert-type means by power means. Rev. Anal. Numér. Théor. Approx. 41(2), 125-129(2012)

13. Hästö, PA: Optimal inequalities between Seiffert's mean and power means. Math. Inequal. Appl. 7(1), 47-53 (2004)

14. Costin, I, Toader, G: Optimal evaluations of some Seiffert-type means by power means. Appl. Math. Comput. 219(9), 4745-4754 (2013)

15. Li, Y-M, Wang, M-K, Chu, Y-M: Sharp power mean bounds for Seiffert mean. Appl. Math. J. Chin. Univ. Ser. B 29(1), 101-107 (2014)

16. Wang, M-K, Qiu, Y-F, Chu, Y-M: Sharp bounds for Seiffert means in terms of Lehmer means. J. Math. Inequal. 4(4), 581-586 (2010)

17. Chu, Y-M, Long, B-Y, Gong, W-M, Song, Y-Q: Sharp bounds for Seiffert and Neuman-Sándor means in terms of generalized logarithmic means. J. Inequal. Appl. 2013, Article ID 10 (2013)

18. Gao, S-Q: Inequalities for the Seiffert's means in terms of the identric mean. J. Math. Sci. Adv. Appl. 10(1-2), 23-31 (2011)

19. Liu, H, Meng, X-J: The optimal convex combination bounds for Seiffert's mean. J. Inequal. Appl. 2011, Article ID $686834(2011)$

20. Jiang, W-D, Qi, F: Some sharp inequalities involving Seiffert and other means and their concise proofs. Math. Inequal. Appl. 15(4), 1007-1017 (2012)

21. Sun, H, Song, Y-Q, Chu, Y-M: Optimal two parameter bounds for the Seiffert mean. J. Appl. Math. 2013, Article ID 438971 (2013)

22. Gong, W-M, Song, Y-Q, Wang, M-K, Chu, Y-M: A sharp double inequality between Seiffert, arithmetic, and geometric means. Abstr. Appl. Anal. 2012, Article ID 684834 (2012)

23. Yang, Z-H, Song, Y-Q, Chu, Y-M: Monotonicity of the ratio of the power and second Seiffert means with applications. Abstr. Appl. Anal. 2014, Article ID 840130 (2014)

24. Sándor, J: On certain inequalities for means III. Arch. Math. 76(1), 30-40 (2001)

25. Jiang, W-D, Cao, J, Qi, F: Two sharp inequalities for bounds the Seiffert mean by the arithmetic, centroidal, and contraharmonic means. arXiv:1201.6432v1 [math.CA]

26. Sándor, J: Über zwei Mittel von Seiffert. Wurzel 36, 104-107 (2002)

\section{Submit your manuscript to a SpringerOpen ${ }^{\circledR}$ journal and benefit from:}

- Convenient online submission

Rigorous peer review

- Immediate publication on acceptance

- Open access: articles freely available online

- High visibility within the field

- Retaining the copyright to your article 